LETTERS TO THE EDITOR

\title{
Evaluation of Short-Term Effectiveness of the Disease Management Program "Di.Pro.Di." on Continuity of Care of Patients with Congestive Heart Failure
}

\author{
Leandro Pecchia, $\mathrm{PhD}$ \\ Department of Biomedical, Electronic and Telecommunication Engineering (DIBET) University \\ Federico II of Naples Naples, Italy \\ Fernando Schiraldi, MD Sossio Verde, MD E. Mirante, MD \\ Intensive Care Unit Public Hospital San Paolo, Azienda Sanitaria Locale Napoli 1 (A.S.L. Na1) \\ Nord Naples, Italy \\ Peter A. Bath, $\mathrm{PhD}$ \\ Department of Information Studies Centre for Health Information Management Research \\ University of Sheffield Sheffield, United Kingdom \\ Marcello Bracale \\ DIBET University Federico II of Naples Naples, Italy
}

The published version of this paper can found at:

http://onlinelibrary.wiley.com/doi/10.1111/j.1532-5415.2010.02985.x/abstract

Pecchia L, Schiraldi F, Verde S, Mirante E, Bath PA and Bracale M, 2010. Evaluation of shortterm effectiveness of the Disease Management Program "Di.Pro.Di." on continuity of care of patients with Congestive Heart Failure. Journal of The American Geriatrics Society, 58(8), 16034, DOI: 10.1111/j.1532-5415.2010.02985.x

To the Editor: This study aimed to assess the early effectiveness of a disease management program (DMP), called "Dimissione Protetta Difficile" (Di.Pro.Di) conducted by personnel from the intensive care unit (ICU) of Public Hospital S. Paolo, Naples, Italy. This hospital serves an area of $31 \lambda \mathrm{km}^{2}$ with 211,000 inhabitants $(20.6 \%$ aged $\geq 65)$. This controlled nonrandomized trial aimed to stabilize patients fully with three home visits in the 3 months after discharge. Rehospitalizations and hospital length of stay of elderly patients suffering from congestive heart failure (CHF) after discharge from the ICU were focused on, because reducing these outcomes is a crucial challenge for developed countries because of their increasing elderly population. The literature shows that DMPs improve care.[1-3] Comparing these outcomes in treatment and control groups, using conventional statistic tests, it was observed, albeit in small numbers, that 
Di.Pro.Di significantly reduces the number and risk of rehospitalizations and total hospital length of stay.

\section{METHODS}

Multidisciplinary teams evaluate patients and educate their families. Patients receive up to three domiciliary visits in the 3 months after discharge. Telephone communication integrates these visits. A physician evaluates the patient's condition and, if there is mild deterioration, modulates the therapy or orders further investigations. If there is major deterioration, the patient is rehospitalized. If the patient health condition is stabile, he is fully discharged.[4] The group of patients enrolled in the program (treatment group, TG) were benchmarked with a group of patients hospitalized in the same structure but not enrolled in the program (control group, CG). The outcomes of a subset of patients enrolled in the TG were retrospectively analyzed. A $t$-test and a chi-square test with Yates' correction were performed to assess the statistical significance of the results and the homogeneity between groups.

\section{Protocol}

Two hundred fifty patients were involved in the Di.Pro.Di, approximately 20 at any one time. Sixteen patients met the inclusion criteria: aged 65 and older, New York Heart Association classification II or III, high risk of rehospitalization, and adequate family support.

The hospital provides the required predosed drugs. During each visit, a gerontologist or cardiologist and a nurse, supported by a car driver or orderly, perform and electrocardiogram, oximetry, blood-gas analysis, capillary blood glucose, and urinalysis.

Oxygen therapy or pulmonary ventilation might also be required. After the third visit, the patient is discharged from the Di.Pro.Di and, according to the stability criteria, rehospitalized or transferred to local health services.

\section{RESULTS}

The results of this study are summarized in Table 1 .

\section{Outcomes}

\section{TG After Di.Pro.Di}

The TG included 16 patients with a mean age of $81.0 \pm 8.8$. Four patients (25\%) were rehospitalized, for a total of four rehospitalizations, (mean 0.3 , maximum of one per patient). The total hospital length of stay was 17 days (mean total $1.1 \pm 2.1$ days per patient).

TG Before Di.Pro.Di 
Six patients in the TG were investigated retrospectively for the year before the Di.Pro.Di. Five of them (83\%) were rehospitalized, for a total of 11 rehospitalizations (mean 1.8 hospitalizations; maximum 4 per patient). Total hospital length of stay for these patients was 69 days (mean total 11.5 \pm 7.2 days per patient).

\section{Control Group}

The CG included 18 patients with a mean age of 79.5 \pm 9.6 . Eleven $(61.1 \%)$ were rehospitalized, for a total of 17 rehospitalizations (mean 0.9, maximum 3 per patient). Total hospital length of stay was 234 days (mean total $13.0 \pm 7.7$ days per patient).

\section{Homogeneity of $T G$ and $C G$}

No statistically significant difference was observed between the CG and TG before Di.Pro.Di in terms of mean age, number of rehospitalizations, and hospital length of stay. In both groups, the number of rehospitalizations and hospital lengths of stay were slightly higher than reported in previous studies, $[5,6]$ possibly because the mean age was slightly higher.

\section{Hospitalizations}

The risk of rehospitalization in the TG was $40 \%$ lower $(\mathrm{p}=0.03)$ than in the CG. The ratio of the risk of rehospitalization, after the Di.Pro.Di and before, was $0.30 \quad(\mathrm{p}=0.009)$.

\section{Length of Stay}

The duration of hospital stay was 10.4 days shorter in the TG (P\&lt;.001) than in the CG. The duration of hospital stay was in the TG was 11.9 days shorter after the Di.Pro.Di than before (P\&lt;.001).

Table 1. Rehospitalizations and Hospital Length of Stay According to Group in the 3 Months After Discharge

\begin{tabular}{|c|c|c|c|}
\hline \multirow[t]{2}{*}{ Outcome } & \multirow{2}{*}{$\begin{array}{l}\text { Control Group } \\
\quad(\mathrm{n}=18)\end{array}$} & \multicolumn{2}{|c|}{ Treatment Group } \\
\hline & & $\begin{array}{c}\text { Before } \\
\text { Di.Pro.Di* }(n=6)\end{array}$ & $\begin{array}{l}\text { After Di.Pro.Di } \\
\quad(\mathrm{n}=16)\end{array}$ \\
\hline \multicolumn{4}{|l|}{ Rehospitalizations, $\mathrm{n}$} \\
\hline Patients rehospitalized & 11 & 5 & 4 \\
\hline Rehospitalizations & 17 & 11 & 4 \\
\hline \multicolumn{4}{|l|}{ Length of hospital stay } \\
\hline Days per group, $\mathrm{n}$ & 234 & 69 & 17 \\
\hline Days per patient, mean \pm standard deviation & $13.0 \pm 7.7$ & $11.5 \pm 7.2$ & $1.1 \pm 2.1$ \\
\hline
\end{tabular}

Di.Pro.Di significantly reduced number of rehospitalizations and hospital length of stay. A possible reason is that Di.Pro.Di allows patients to be fully stabilized before complete discharge. 
As in other DMPs, Di.Pro.Di improved the education of patients and families, improving adherence to therapy and lifestyle after discharge. These preliminary results suggest that Di.Pro.Di. improves the effectiveness of care for elderly patients with CHF.

\section{ACKNOWLEDGMENTS}

Conflict of Interest: The editor in chief has reviewed the conflict of interest checklist provided by the authors and has determined that the authors have no financial or any other kind of personal conflicts with this paper.

Author Contributions: L. Pecchia: study concept and design, analysis and interpretation of data, and preparation of manuscript. F. Schiraldi: study concept and design, trial supervision, acquisition of subjects and data, interpretation of data, and preparation of manuscript. S. Verde: study concept and design, acquisition of subjects and data, analysis and interpretation of data, and preparation of manuscript. E. Mirante: study concept and design and acquisition of subjects and data. P. Bath: data presentation and preparation of manuscript. M. Bracale: study concept and design, supervision of analysis and interpretation of data, and preparation of manuscript.

Sponsor's Role: None.

\section{REFERENCES}

1. Gonseth J, Guallar-Castillon P, Banegas JR et al. The effectiveness of disease management programmes in reducing hospital re-admission in older patients with heart failure: A systematic review and meta-analysis of published reports. Eur Heart J 2004;25:1570-1595.

2. Holland R, Battersby J, Harvey I et al. Systematic review of multidisciplinary interventions in heart failure. Heart 2005;91:899-906.

3. Pecchia L, Bracale U, Bracale M. Health Technology Assessment of Home Monitoring for the Continuity of Care of Patient Suffering from Congestive Heart Failure. World Congress on Medical Physics and Biomedical Engineering, September 7-12, 2009, Munich, Germany: 184187.

4. Dickstein K, Cohen-Solal A, Filippatos G et al. Guidelines for the diagnosis and treatment of acute and chronic heart failure. Eur J Heart Failure 2008;10:933-989.

5. Guder G, Frantz S, Bauersachs J et al. Reverse epidemiology in systolic and nonsystolic heart failure cumulative prognostic benefit of classical cardiovascular risk factors. Circul Heart Fail 2009;2:563-571.

6. Harjai KJ, Thompson HW, Turgut Tet al. Simple clinical variables are markers of the propensity for readmission in patients hospitalized with heart failure. Am J Cardiol 2001;87:234237. 